\title{
Universal role of migration in the evolution of cooperation
}

\author{
YANG HanXin ${ }^{1} \&$ WANG BingHong ${ }^{1,2 *}$ \\ ${ }^{1}$ Department of Modern Physics, University of Science and Technology of China, Hefei 230026, China; \\ ${ }^{2}$ Research Center for Complex System Science, University of Shanghai for Science and Technology and Shanghai Academy of System Science, \\ Shanghai 200093, China
}

Received May 23, 2011; accepted July 11, 2011

\begin{abstract}
We study the role of unbiased migration in cooperation in the framework of the spatial evolutionary game on a variety of spatial structures, namely a regular lattice, continuous plane and complex networks. A striking finding is that migration plays a universal role in cooperation, regardless of the spatial structure. For a high degree of migration, cooperators cannot survive owing to their failure to form cooperator clusters that resist attacks by defectors. Meanwhile, for a low degree of migration, cooperation is considerably enhanced relative to that in the static spatial game, which is due to the strengthening of the boundaries of cooperator clusters by the occasional accumulation of cooperators along the boundaries. The cooperator cluster thus becomes more robust than that in the static game and defectors near the boundary can be assimilated by cooperators. The cooperator cluster thus expands, which facilitates cooperation. The general role of migration will be substantiated by sufficient simulations relating to heuristic explanations.
\end{abstract}

evolutionary game, cooperation, migration

Citation: $\quad$ Yang H X, Wang B H. Universal role of migration in the evolution of cooperation. Chinese Sci Bull, 2011, 56: 3693-3696, doi: 10.1007/s11434-011-4768-5

Cooperation is fundamental to biological and social systems. Understanding factors that facilitate and hamper cooperation is a significant issue. In the framework of evolutionary games, a number of mechanisms in favor of cooperation have been found, such as costly punishment [1,2], reputation [3,4] and social diversity [5-7]. Quite recently, the role of migration in cooperative behavior has drawn growing interest [8-14] because of the fact that migration is a common feature in nature and society [15-17]. For example, millions of animals migrate in the savannas of Africa every year, and thousands of people travel among countries every day. In this regard, Vainstein et al. [10] considered a scenario that individuals can move to neighboring sites on a two-dimensional lattice randomly with some probability. In particular, it was found that such movement can maintain and even enhance cooperation. More recently, Meloni et al. [13] studied the evolutionary games involving mobile players on a continuous plane. Their results showed that cooper-

*Corresponding author (email: bhwang@ustc.edu.cn) ation can survive provided that both the temptation to defect and the velocity at which individuals move are not too high. Beyond random migration, Helbing and $\mathrm{Yu}$ [11] proposed a success-driven migration strategy that is spurred by the pursuit of profit as a nature of individuals. Specifically, individuals tend to move to neighboring sites with the highest estimated payoffs. Interestingly, such migration results in an outbreak of cooperation in a noisy environment. Recently, Yang et al. [14] proposed an aspiration-induced migration in which individuals migrate to new sites provided that their payoffs are below some aspiration level. It is found that a moderate aspiration level best favors cooperative behavior.

Although it has been demonstrated that migration can promote cooperation in evolutionary games on some regular spatial structures [10-12], the role of migration on other kinds of structures, for instance, complex networks, is unknown. A natural concern is then whether migration plays a general role in cooperation, regardless of the underlying structures, or the role of migration is dependent on the structures. To address this issue, this paper incorporates 
random migration in evolutionary games on a variety of spatial structures, involving continuous space, regular structure, and typical complex topologies. Strikingly, we find that the role of random migration in promoting cooperation is universal, regardless of structures. This is somewhat counterintuitive in the sense that the mobility of individuals may weaken the stability of cooperation clusters, which is key for the survival of cooperators. However, we will substantiate the positive effect of random migration on cooperation with intensive simulations and provide convincing explanations of the underlying mechanisms.

\section{Model}

To be concrete, we use the Prisoner's Dilemma [18] in our research. In principle, the Prisoner's Dilemma is a game played by two players, each of whom chooses one of two strategies, cooperation or defection. They both receive payoff $R$ upon mutual cooperation and $P$ upon mutual defection. If one defects while the other cooperates, the cooperator receives $S$ while the defector gets $T$. The ranking of the four payoff values is $T>R>P>S$. Thus, in a single round of the Prisoner's Dilemma, it is best to defect regardless of the opponent's decision. The Prisoner's Dilemma has attracted much attention in theoretical and experimental studies of cooperative behavior. Following common practice [16], we set $T=b(1<b<2), R=1$, and $P=S=0$, where $b$ represents the temptation to defect.

To explore the role of migration, we resort to the spatial game in which individuals are placed on spatial structures. Since the introduction of spatial structures into evolutionary games by Nowak and May [19], there has been much interest in revealing the effects of population structures on cooperation, ranging from regular lattices to complex networks. In spatial games, interactions among individuals are restricted to immediate neighbors and usually neighbors of an arbitrary individual remain fixed. Meanwhile, in the presence of migration, neighboring individuals can be changed by encountering different partners as time goes on. In the seminal works of Vainstein et al. [10] and Meloni et al. [13], random migration was considered in spatial games on a two-dimensional lattice and on a continuous plane, respectively. Inspired by this original research, we extend migration on a regular structure to complex networks and uncover the general role of migration in promoting and hampering cooperation.

\section{Simulation results}

Let us first consider individuals moving on a continuous square plane with periodic boundary conditions. Initially, individuals are randomly located on a square plane and cooperators and defectors with equal percentage are randomly distributed on the plane. At each time step, each individual plays the game with individuals falling within a circle of radius $q$ centered at his/her current position. Individuals synchronously update their strategies according to a best-takes-over reproduction; that is, each individual compares his/her payoff with that of his/her neighbors and updates his/her strategy by following the one (including himself/herself) with the greatest payoff. After the strategy updating process, individuals move to new locations with random directions of motion at migration speed $v$. The absolute value of $v$ is the distance an individual can move in a typical time step. Figure 1(a) shows the fraction of cooperators as a function of the temptation to defect for different values of migration speed $v$. We see that compared with the static case $(v=0)$, cooperation is enhanced in a wide range of temptation to defect when individuals move slowly ( $v=$ $0.04)$. On the other hand, fast motion $(v=1)$ leads to complete extinction of cooperators, analogous to the situation arising in the well-mixed population.
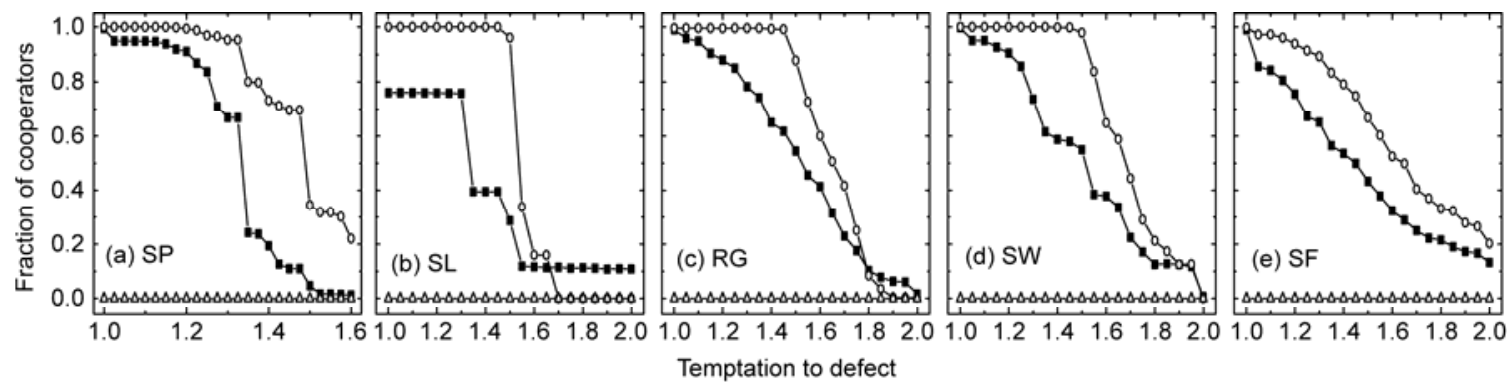

Figure 1 Fraction of cooperators as a function of the temptation to defect $b$. (a) Individuals are located on a continuous square plane (SP) of linear size $L=$ 20 with periodic boundary conditions. Interaction radius $q=1$. Filled squares, open circles and open triangles show results for $v=0$, $v=0.04$ and $v=1$, respectively. Lines are visual guides. (b)-(e) Individuals are located on square lattices (SL), random graphs (RG), small-world networks (SW) and scale-free networks (SF). Average connectivity $z=4$ for SL and SF, $z=6$ for RG and SW. Filled squares, open circles and open triangles show results for $p=0$, $p=$ 0.001 and $p=1$, respectively. The population size is 1024 . The equilibrium fraction of cooperators is obtained by averaging over $10^{5}$ time steps after a transient period of $10^{5}$ time steps. Each data point depicted corresponds to an average of more than 1000 simulations; that is, 100 runs for 10 different realizations of the same class of graph. 
Next, we study individuals migrating on various network models, including square lattices (SL), random graphs (RG) [20], small-world networks (SW) [21] and scale-free networks (SF) [22]. Initially, each node of the network is occupied by an individual, and individuals with two strategies (cooperators or defectors) are randomly distributed. At each time step, each individual plays the game with individuals sitting on the same node and neighboring nodes. Individuals synchronously update their strategies according to best-takes-over reproduction and then each individual jumps to a randomly chosen neighboring node with probability $p$ (a node can be occupied by more than one individual). Results are shown in Figure 1(b)-(e). As compared with the static case $(p=0)$, low migration probabilities $(p=0.001)$ promote cooperation in a wide range of the temptation to defect $b$ (except for large $b$ on square lattices and random graphs, where the fraction of cooperators is lower than that for $p=0$ ), similar to the results for the continuous plane. Meanwhile, for high migration probability $(p=1)$, defectors dominate the whole population.

From Figure 1, we find by comparison with the spatial game in the absence of migration, that low migration speeds/probabilities can considerably promote cooperation whereas high migration speed/probability facilitates defection, which regardless of underlying structures in a qualitative sense. We also investigated the dependence of the fraction of cooperators on the migration speed $v$ and probability $p$ by fixing the value of temptation to defect $b$. As exhibited in Figure 2, as $v$ and $p$ increase, the fraction of cooperation monotonously decreases. It is known that in spatial games, cooperators can survive by forming clusters [23,24], for which the benefits of mutual cooperation can outweigh losses against defectors, thus maintaining cooperation. Combining Figures 1 and 2, we find that the effect of migration on cooperation is twofold. For high migration speed/probability, cooperation is inhibited since cooperator clusters hardly form with the frequent change of neighbors. Without the protection of cluster structures, a cooperator can hardly survive. For a low degree of migration, it is not easy to determine the effect of migration on cooperation. A heuristic explanation is that after the construction of cooperator clusters, a small perturbation along the boundary due to migration can trigger the expansion of cooperator clusters and enhance the fraction of cooperation.

To intuitively understand the effect of perturbation around the cooperator cluster on cooperation, we construct a crossed cooperator cluster (comprising five cooperators) surrounded by defectors on a square lattice (Figure 3 ). The temptation to defect $b=1.4$. If individuals are immobile, the crossed cooperator cluster is stable and remains unchanged. In the presence of migration, situations arising at the cluster boundary can be classified into four types: (1) a cooperator at the boundary enters the defector cluster, (2) a defector at the boundary intrudes into the cooperator cluster, (3) a defector moves away from the boundary within its defector

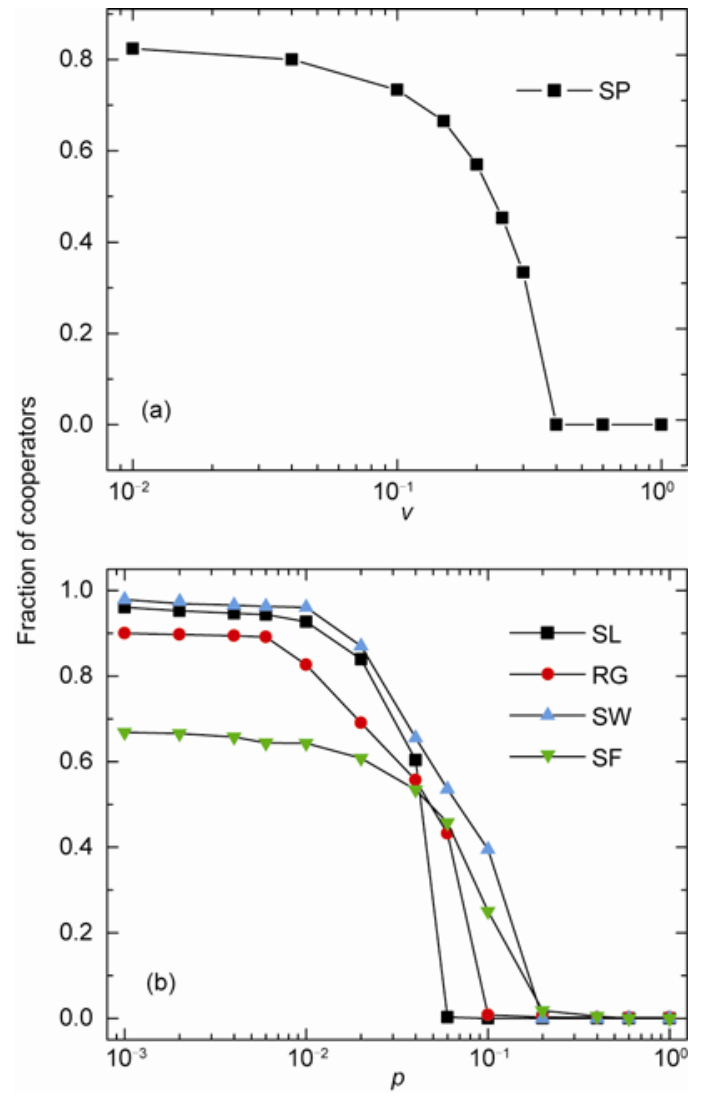

Figure 2 Fraction of cooperators as a function of the migration speed $v$ or the migration probability $p$. (a) Individuals migrate on a square plane (SP) of linear size $L=20$. The temptation to defect $b=1.35$ and the interaction radius $q=1$. (b) Individuals migrate on various networks. The temptation to defect $b=1.5$. Average connectivity $z=4$ for SL and SF, $z=6$ for RG and SW. The population size is 1024 . Each data point depicted corresponds to an average of more than 1000 simulations; that is, 100 runs for 10 different realizations of the same class of graph.

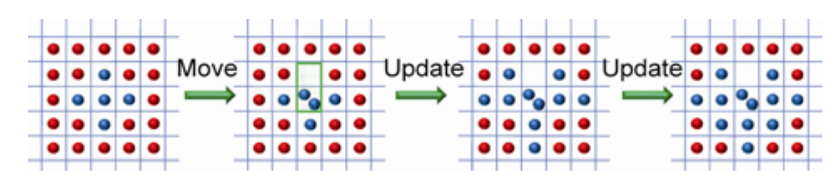

Figure 3 Red balls represent defectors and blue balls denote cooperators. Each individual plays the game with individuals who are located on the same lattice and the neighboring lattices. The temptation to defect $b=1.4$. As a cooperator moves from the cluster boundary into the core of a cooperator cluster, the cooperator cluster expands.

cluster; and (4) a cooperator moves away from the boundary within its cooperator cluster. In case (1), the irruptive cooperator changes to a defector; in case (2), the irruptive defector changes to a cooperator; in case (3), nothing happens. Cases (1) to (3) do not drastically affect the fraction of cooperators in the system (data not shown here). However, in case (4), the territory of the cooperator cluster expands to other regions of the square lattice and the number of cooperators increases from 5 to 12, as shown in Figure 3. It is thus the arising of case (4) that promotes the prevalence of cooperation in the population. In general, this scenario is 
representative of the strengthening of the cooperator cluster boundary by multiple cooperators at the same node (i.e. the density of cooperators is augmented along the boundary). A direct result is that the payoffs for cooperators along the boundary increase and defectors near the boundary are assimilated. As a result, cooperator clusters expand and cooperation is enhanced.

To visually observe how a low degree of migration affects the evolution of cooperator clusters and defector clusters, we initially set cooperators in the middle region of a square plane, while defectors are located in other regions. Figure 4(a)-(c) show that, for low migration speed, the cooperator cluster gradually expands as time step $t$ increases and cooperators dominate the whole population in the end. For the static case in which individuals do not move, the cooperator cluster remains almost unchanged (Figure 4(d)).

\section{Discussion and conclusion}

In summary, we have studied the role of migration in cooperation in the framework of the spatial Prisoner's Dilemma game on a variety of spatial structures. Our finding is that although a high degree of migration results in the extinction of cooperation, a low degree of migration considerably enhances cooperation by increasing the cooperator density along the boundary of the cooperator clusters. Owing to the accumulation of cooperators along the boundary, the benefits of mutual cooperation outweigh losses against defectors near the boundary; this thus not only allows cooperation within the cluster to be maintained, but also induces the expansion of the cooperator cluster, in contrast to the case for the static spatial game. The strengthening at the boundary of cooperator clusters induced by the small degree of migration plays a key role in the enhancement of cooperation, regardless of the underlying structure on which the evolutionary game takes place.

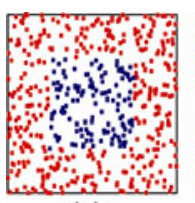

(a)

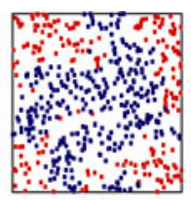

(b)

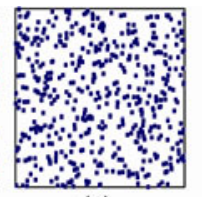

(c)

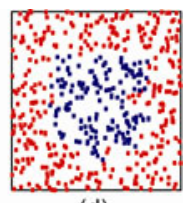

(d)
Figure 4 Snapshots of distributions of cooperators (blue) and defectors (red) on a square plane of linear size $L=20$. Initially, we set individuals located in the middle region of the plane as cooperators (the cooperative region is a $10 \times 10$ square), while defectors are located in other regions. The population size is 500, the interaction radius $q=1$ and the temptation to defect $b=1.5$. (a)-(c) Snapshots at different time steps $t$ for $v=0.1$. (a) $t$ $=0$, (b) $t=263$ and (c) $t=1315$. (d) The snapshot for $v=0$ when the system reaches equilibrium.
This work was supported by the National Basic Research Program of China (2006CB705500), the National Important Research Project (91024026), the National Natural Science Foundation of China (10975126, 10635040, 11005001) and the Specialized Research Fund for the Doctoral Program of Higher Education of China (20093402110032).

1 Hauert C, Traulsen A, Brandt H, et al. Via freedom to coercion: The emergence of costly punishment. Science, 2007, 316: 1905-1907

2 Ohtsuki H, Iwasa Y, Nowak M A. Indirect reciprocity provides only a narrow margin of efficiency for costly punishment. Nature, 2009, 457: 79-82

3 Nowak M A, Sigmund K. Evolution of indirect reciprocity by image scoring. Nature, 1998, 393: 573-577

4 Ohtsuki H, Iwasa Y. How should we define goodness? - Reputation dynamics in indirect reciprocity. J Theor Biol, 2004, 231: 107-120

5 Santos F C, Santos M D, Pacheco J M. Social diversity promotes cooperation in public goods games. Nature, 2008, 454: 213-216

6 Perc M, Szolnoki A. Social diversity and promotion of cooperation in the spatial prisoner's dilemma game. Phys Rev E, 2008, 77: 011904

7 Yang H X, Wang W X, Wu Z X, et al. Diversity-optimized cooperation on complex networks. Phys Rev E, 2009, 79: 056107

8 Enquist M, Leimar O. The evolution of cooperation in mobile organism. Anim Behav, 1993, 45: 747-757

9 Aktipis C A. Know when to walk away: Contingent movement and the evolution of cooperation. J Theor Biol, 2004, 231: 249-260

10 Vainstein M H, Silva A T C, Arenzon J J. Does mobility decrease cooperation? J Theor Biol, 2007, 244: 722-728

11 Helbing D, Yu W. The outbreak of cooperation among success-driven individuals under noisy conditions. Proc Natl Acad Sci USA, 2009, 106: 3680-3685

12 Sicardi E A, Fort H, Vainstein M H, et al. Random mobility and spatial structure often enhance cooperation. J Theor Biol, 2009, 256: 240-246

13 Meloni S, Buscarino A, Fortuna L, et al. Effects of mobility in a population of prisoner's dilemma players. Phys Rev E, 2009, 79: 067101

14 Yang H X, Wu Z X, Wang B H. Role of aspiration-induced migration in cooperation. Phys Rev E, 2010, 81: 065101

15 Wang J Z, Lin G H, Huang J H, et al. Applications of stable isotopes to study plant-animal relationships in terrestrial ecosystems. Chinese Sci Bull, 2004, 49: 2339-2347

16 Mi J K, Xiao X M, Liu D H, et al. An investigation of water-gas interface migration of the upper Paleozoic gas pool of the Ordos Basin using reservoir fluid inclusion information. Chinese Sci Bull, 2004, 49: 735-739

17 Liu H, Guo G Q, He Y K, et al. Nuclear migration: Endless efforts toward unraveling its molecular apparatus. Chinese Sci Bull, 2003, 48: 615-619

18 Axelrod R, Hamilton W D. The evolution of cooperation. Science, 1981, 211: 1390-1396

19 Nowak M A, May R M. Evolutionary games and spatial chaos. Nature, 1992, 359: 826-829

20 Erdos P, Renyi A. On the evolution of random graphs. Publ Math Debrecen, 1959, 6: 290-297

21 Newman M E J, Watts D J. Renormalization group analysis of the small-world network model. Phys Lett A, 1999, 263: 341-346

22 Barabasi A L, Albert R. Emergence of scaling in random networks. Science, 1999, 286: 509-512

23 Szabo G, Toke C. Evolutionary prisoner's dilemma game on a square lattice. Phys Rev E, 1998, 58: 69-73

24 Gomez-Gardenes J, Campillo M, Floria L M, et al. Dynamical organization of cooperation in complex topologies. Phys Rev Lett, 2007, 98: 108103

Open Access This article is distributed under the terms of the Creative Commons Attribution License which permits any use, distribution, and reproduction in any medium, provided the original author(s) and source are credited. 